\title{
The Effectiveness of Virtual Clinical Excursion among Pre-Licensure Nursing Students
}

\author{
Alham Abuatiq ${ }^{1 *}$ and Claudia Davis ${ }^{2}$ \\ ${ }^{1}$ College of Nursing, South Dakota State University, USA \\ ${ }^{2}$ California State University San Bernardino, USA \\ *Corresponding author: College of Nursing, South Dakota State University, Brookings, SD, USA \\ Submission: 啙 February 25, 2018; Published: 監 May 22, 2018
}

\begin{abstract}
Background: The aims of this study were to first, investigate nursing students' perceptions about the

effectiveness of implementing the Virtual Clinical Excursions (VCE) in improving their learning experiences. Second, to evaluate the effectiveness of VCE in improving students' physical assessment skills. Third, to investigate if the VCE improved students' oral and written communication skills.

Methodology: Mixed methods descriptive design, sample size was forty-two students.

Results: VCE effects on improving students' physical assessment skills were evaluated by using Likert type question from $0=$ "Not applicable" to $5=$ "Strongly agree".26.2\% ( $n=11)$ of students reported that the VCE was very effective in improving their nursing learning experiences. Findings reflected that $23.8 \%(\mathrm{n}=10)$ reported "Strongly agree", and $50 \%(\mathrm{n}=21)$ reported "Agree" in response to the VCE having improved their physical assessment skills. The majority of students $45.2 \%(n=19)$ reported, "Agree" that (VCE) improved their written communication skills, versus $40.5 \%$ (n= 17) of students reported "Neutral" for the (VCE) effects on improving their oral communication skills.
\end{abstract}

Conclusion: Overall, students reported that the VCE provided a digital interaction with virtual patient's scenarios that was effective in improving their learning experiences

Keywords: Virtual clinical excursions; Student nurse experience; E-learning; Undergraduate student nurses; Virtual clinical

\section{Introduction}

Over the past few years, enrollment in baccalaureate, master's, and doctoral nursing programs has increased dramatically [1]. In addition, the high demand for nurses, coupled with the increasing number of students enrolled in the nursing programs nationwide, creates a challenge for nursing faculty to find clinical training facilities for students. High volume of student enrollment, and the ascending number of online nursing programs increased the demand to use online teaching methodologies in nursing curriculums. Virtual learning environments may provide a viable solution to accommodate the increased volume of student's enrollment in nursing programs, and provide individualized selfpaced evaluation for student's performance. While the necessity is evident, there appears to be a paucity of research investigating student's experience of virtual clinical environment. To that end, there is a need to investigate the effectiveness of using VCE on students' learning experiences to integrate virtual e-learning programs with virtual patient's interaction as a learning venue for their pre-licensure nursing students. Thus, the aims of this study are to first, investigate nursing students' perceptions about the effectiveness of implementing the VCE. Second, to evaluate the effectiveness of VCE in improving students' assessment skills, and third, to investigate if the VCE improved students' oral and written communication skills [2].

\section{Background}

Virtual Clinical Excursions (VCE) is an online e-learning system that simulates the healthcare setting through a virtual hospital in which students can provide basic nursing care to virtual patients. This e-learning program provides a virtually safe, individualized, and computer evaluated virtual training for students. Students can navigate in the virtual hospital and learn basic nursing skills including physical assessment, check labs, medication administration, and online drug resources along with videos reflecting patient-nurse communication skills.

\section{Literature Review}

Finding a clinical hospital setting to train and educate nursing students is a constant challenge for many nursing schools, many teaching hospitals are inundated with requests from multiple schools requesting student clinical training. One of the obstacles that face the pre-licensure nursing programs is the lack of inpatient clinical sites to prepare students for nursing practice. Factors that 
contribute to this worsening problem include nursing shortage, faculty shortage, and lack of collaboration between nurses in academic and practice settings and with other healthcare professionals, cost, and safety and liability issues [3].

In 2012, many qualified applicants for the baccalaureate and graduate nursing programs were not admitted by four-year colleges and universities [1] This phenomenon is supported by a survey reported by the AACN that found 75,587 qualified applications to nursing schools were not accepted in 2011 due to primarily a shortage of faculty and resource constraints [1]. Accordingly, several reasons reported by nursing schools for not accepting all qualified students into entry-level baccalaureate programs, include insufficient clinical teaching sites (65.2\%), a lack of faculty $(62.5 \%)$, limited classroom space (46.1\%), insufficient preceptors $(29.4 \%)$, and budget cuts $(24.8 \%)$ [1]. These factors may have an adverse effect on the pre licensure student's learning experience.

\section{Impact of virtual e-learning on nursing education}

Some nursing programs use several types of e-learning systems to deal with the high volume of students and shortage of faculty to teach courses especially clinical based courses. The virtual clinical excursions provide a virtual hospital setting for students in which they can interact, and critically think about their virtual patients' cases. Since the simulation lab is condensed with students most of the time, VCE have the potential to offer virtual space with individualized learning experience for the students.

Early studies involving virtual learning systems have shown positive relationships between implementation, perceived benefits and evidence that this learning approach is engaging. Early evidence also suggests greater benefits among underrepresented minority students $[4,5]$.

Researchers conducted a pilot study that used a one group, post-test survey design to explore senior nursing students' satisfaction with a simulated VCE [6]. Participants in the pilot study completed the online Satisfaction with VCE Survey (SVCES). The SVCES assessed students' evaluation of user-friendliness, realism, knowledge, and quality of the VCE resource. Findings reflected that Millennial and Gen X'ers did not report different SVCES scores, however, students' ratings on eight SVCES items were positively and strongly correlated with computer competence. The authors determined that VCE may be a helpful teaching tool to assist students to acquire information from sources to plan patient care in clinical practice. The researchers recommended that faculty should integrate VCE resources within the nursing curriculum [6]. Accordingly, VCE introduces nursing students to the e-learning approach to improve their confidence and their decision-making strategies. The authors recommended that nursing students should have independent VCE learning scheduled as a mandatory course component. Moreover, students should have tutorial sessions to learn navigation in the VCE in order to decrease any anxiety related to the use of technology in the learning process [6]
The utilization of Virtual Communities allowed faculty to utilize multidimensional experiences that students can use beyond the textbook materials [7]. Constant use of the virtual cases over time is similar to having the opportunity to care for a patient for several days in a row. The Virtual Communities provides faculty with a tool that encourages the development of integrative teaching strategies suggesting that this type of integrative pedagogy is effective for teaching in a practice profession. More importantly, this type of tool seems to facilitate an integrative and contextual approach to teaching, without being overly taxing for the user [7].

By implementing the VCE as an integral assignment in the theory course, the students will have an opportunity to use a virtual hospital setting, meet virtual patients with different diseases, and check their medical charts, lab results, and medications administrations report. VCE may improve a student's decision making abilities, and provide safe evidenced base care to virtual patients in a virtual healthcare setting.

\section{Methodology}

\section{Design}

A cross-sectional design was used for this study. Convenience sampling of 3rd quarter nursing students during the Spring quarter (2015) was utilized. The virtual clinical excursions (VCE) was integrated in the theory course during the last hour of the lecture; the students were assigned to a virtual patient's case synchronized with the theory content. Data was collected via a one-time face to face electronic survey with Likert type questions, numeric scale and open-ended questions.

\section{Ethical considerations}

This study was approved by the university Institutional Review Board. Written and verbal information was given about the study. Participants were informed of the purpose of the study. Questions were answered. Consent forms were signed, and collected from those who voluntarily and anonymously participated in this study.

\section{Sample and setting}

All students who were enrolled in the intermediate medical surgical nursing theory course was eligible to participate in the study. This study was conducted in one of the large public university in California, in one of the Bachelor of Science degree nursing programs, for third quarter nursing students. The students had the theoretical based lecture time for 2 hours, 1 hour for discussion and in the remaining 1 hour were assigned to a selected VCE virtual patient's case related to the theoretical content of the lecture. Using the VCE, students were able to conduct virtual head to toe physical assessment, check patient's electronic medical record, laboratory values, and administer prescribed medications virtually. Students had internet connection via campus. Students had online access the virtual patients' $24 / 7$, with full access to virtual patient's medical records, virtually demonstrate nursing skills including head to toe assessment, and medication administration, along with online 
drug book access present in the nursing station within the virtual hospital.

\section{Inclusion criteria}

The inclusion criteria for the sample included the following; current nursing student in the nursing program, enrollment in the theory medical surgical course at the time of the study, and be able to understand, write, and speak English, and finally, have basic computer skills.

\section{Recruitment}

An informational presentation was provided, and additional teaching case studies were provided for students who did not want to participate. An orientation session to VCE was given to students during the first- class session, and time was given to answer students' concerns and questions. All students were registered in the course and were students pre-license program and were made aware of the study. Students enrolled in the theoretical based course were invited to participate. Detailed instructions were given to students on how to maneuver in the virtual hospital, the instructions guide was taken from the evolve resources provided to students on how to use the virtual clinical excursions VCE. The VCE was integrated in the theory course during the last hour of the lecture; the students were assigned to a virtual patient's case synchronized with the theory content. This study was held in the spring quarter within an eleven-week time frame, and by the end of the spring quarter the students were given a survey with the Likert scale, slide numeric scale, and an open-ended question format to collect data about their perceptions via online survey.

\section{Procedures}

Students were informed that the electronic questionnaire should take approximately 15 minutes. Students took the survey in the classroom setting using their own computers. The prerequisite knowledge and skills students need to have before participating in this study included the following: First, nursing students needed to first have an account and access to Evolve online learning resourceswww.evolve.elsevier.com. A pre-purchased online access the VCE virtual hospital was provided to students in the theory medical surgical class. Students used their own computer, tablet or smart phone to log in to the clinical virtual excursions. In the first session, the students were given an orientation to the virtual hospital provided by VCE. Second, after orientation, students were assigned to a virtual patient's case, and developed competency to maneuver in the virtual hospital setting to assess virtual patients, checked the electronic medical records, checked patients' labs, check the virtual medication room, and learn how to administer prescribed medications virtually.

\section{Measurements}

We developed an electronic survey to collect demographic data and Likert questions consisting of student's perceptions of using the VCE. Numeric scales from $0=$ (not effective) to 10 (very effective) scale questions, Likert type questions items range from not applicable $=0$, Strongly disagree $=1$, Disagree $=2$, Neutral $=3$, Agree $=4$, Strong agree $=5$ were utilized. $A$ total of 28 questions were provided.

\section{Data analysis}

Descriptive statistics including means and standard deviations were calculated representing socio demographic data, e.g. age, gender, and ethnicity. Power analysis was calculated by using G Power 3.1 with P value of 0.05 , for T-test, Power $=0.95$, Correlation Bi-serial model, and effect size is 0.70 the calculated sample size was minimum 16 subjects. Data analysis was done using SPSS 22with significance level of $p<0.05$, findings are presented according to the proposed research aims.

\section{Results}

Socio-demographically, close to half of our students were selfidentified as Hispanic as shown in (Table 1). Approximately, twenty one percent and nineteen percent self-identified as Caucasian and Asian, respectively. Eleven percent of the students identified as other inclusive of multi ethnical groups. Quite typical in the profession of nursing, most of the students were females (73\%) and $(17 \%)$ were males. Mean age was 23 years old (Table 1) represented demographic characteristics of students.

Table 1: Demographic Characteristics of Students.

\begin{tabular}{|c|c|c|c|}
\hline Characteristic & $\mathbf{X}$ & SD & Range \\
\hline Age & 23.1 & 5.33 & $19-46$ \\
\hline Ethnicity & $\mathrm{N}$ & $\%$ & \\
\hline Hispanic & 20 & 47.6 & \\
\hline Caucasian & 9 & 21.4 & \\
\hline Asian & 8 & 19 & \\
\hline Other & 5 & 11 & \\
\hline Gender & & & \\
\hline Female & 34 & $73 \%$ & \\
\hline Males & 8 & $17 \%$ & \\
\hline
\end{tabular}

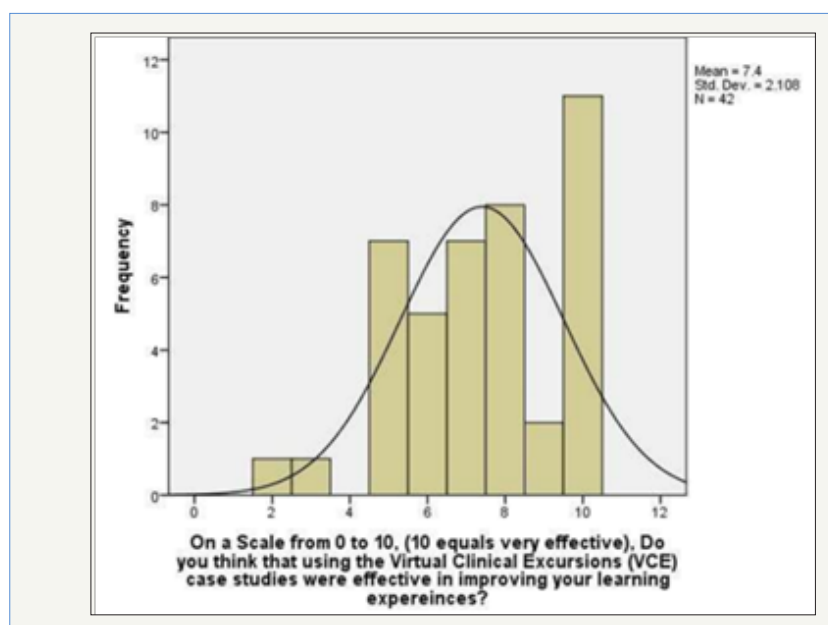

Figure 1: Students Rating for the Effectiveness of VCE on a Scale from 0 to 10 . 
For aim (1) focused on investigating nursing students' perceptions about the effectiveness of implementing the Virtual Clinical Excursions (VCE) in improving their learning experiences. On a scale from $0-10$, with 10 being most effective, the respondents (26\%) indicated that VCE was very effective in improving their learning experiences (Figure 1). Moreover, students were asked to numerically report from $0-10$ scale to report if they do like the use of VCE e-learning system in the nursing program; $28.6 \%(n=12)$ reported score of 10 . For the variable, "Did the VCE meet your nursing learning needs?" $23.8 \%(n=10)$ reported score of 8 and 7 for this variable. (Figure 2) represents frequencies about the VCE rating in meeting students learning needs.

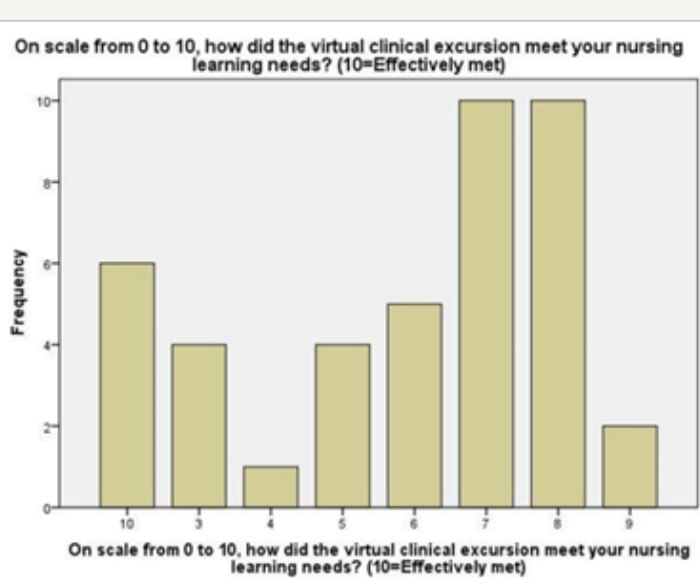

Figure 2: Frequencies for "Did the VCE meet your nursing learning needs?".

With regards to the incorporation of e-learning in nursing program, students were asked to rate this question "In general, do you agree on the use of e-learning in the nursing program $(10=$ strongly agree)". Specifically, results reflected that $33.3 \%$ $(n=14)$ of students reported "Strongly Agree (10)" for the use of

Table 2: Likert Type Questions Highly Ranked by Students. e-learning in nursing, followed by $21.4 \%$ (n=9) who reported "Agree (8)" for the same variable. Figure 3 shows descriptive frequencies for this variable.

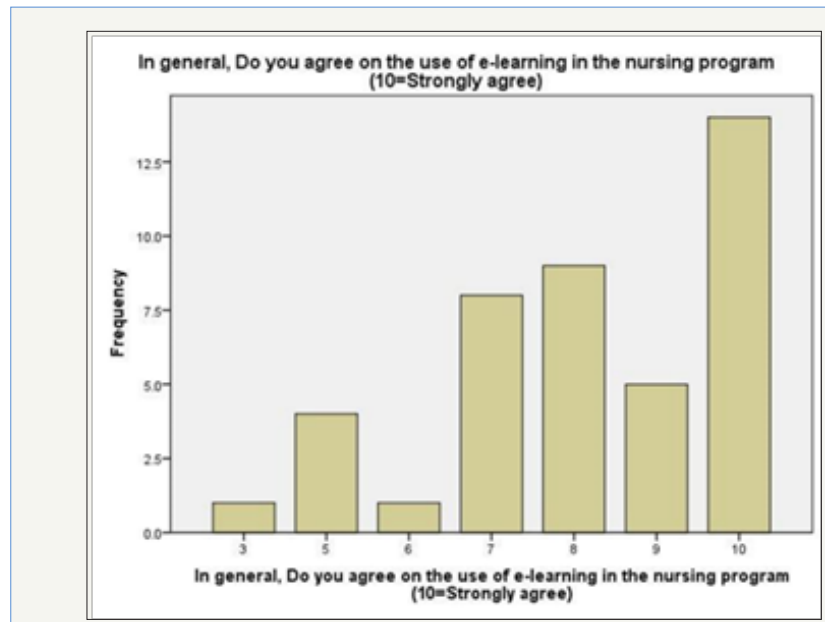

Figure 3: Frequencies for Student's Agreeability about E-Learning in Nursing.

In addition, (Table 2) reports the top eight variables highly ranked in the survey with Likert scale of $0=$ not applicable, $1=$ Strongly Disagree, 2=Disagree, $3=$ Neutral, $4=$ Agree, $5=$ strongly agree type of questions. Descriptive data analysis reflected that the top-rated items included the following: $45.2 \%(n=19)$ of students reported, "Strongly agree" for item \#16, "I was able to check my patient's lab results and correlate it to my patient's medical diagnosis". Followed by $40.5 \%$ (n=17) of students reported "Strongly Agree" for item \#17, "I was able to virtually administer medication for my patient according to the Medication Administration Record". Along with item \#18, "I was able to check medications information from the drug book icon". In addition, 35.7\% (n=15) of students reported, "Strongly agree "for item \#15, "I was able to select and prioritize Nursing diagnosis for my patient".

\begin{tabular}{|c|c|c|c|c|}
\hline Rank & Item & & Frequency \% & $\mathbf{n}$ \\
\hline & \# & & "Strongly Agree" & \\
\hline $1 \mathrm{st}$ & 16 & I was able to check my patient's lab results and correlate it to my patient's medical diagnosis & $45.20 \%$ & 19 \\
\hline 2nd & 17 & $\begin{array}{l}\text { I was able to virtually administer medication for my patient according to the Medication } \\
\text { Administration Record }\end{array}$ & $40.50 \%$ & 17 \\
\hline 3rd & 18 & I was able to check medications information from the drug book icon & $40.50 \%$ & 17 \\
\hline 4 th & 5 & The e-learning system helped me implement the nursing process & $35.70 \%$ & 15 \\
\hline 5 th & 12 & I was able to virtually take vital signs for my patient, and implement interventions accordingly & $28.60 \%$ & 12 \\
\hline 6th & 28 & I was able to improve my documentation skills & $28.60 \%$ & 12 \\
\hline 7 th & 19 & I developed more competency in pharmacology & $26.20 \%$ & 11 \\
\hline 8th & 14 & I was able to virtually implement nursing interventions to my patient & $26.20 \%$ & 11 \\
\hline
\end{tabular}


Participants answered the open-ended questions, and the following are some selected students' answers related to this question: "Did the training on Virtual Clinical Excursions meet your nursing learning needs? And how?" Student \#11 reported "E-learning is an immensely helpful tool both in the class \& outside. E-learning creates a light-weight versatile approach to the nursing process \& learning."Student \#14: "Lecture can easily be tuned out, but e-learning is very interactive, so it helps engage the students more. We should use it more."Student \# 20: "I think it does help in some ways such as learning what labs are out of the normal range and what medications they are taking."Student \#36:"It is a great way to connect many materials, including pharmacology and medical surgical and pathophysiology. It is a great way to make the connections."

\section{Discussion}

VCE effects on improving student's physical assessment skills were evaluated by using Likert type questions from $0=$ "not applicable" to $5=$ "strongly agree". Findings reflected that $23.8 \%$ (n= $10)$ reported "Strongly agree", and $50 \%(n=21)$ reported "Agree" to responses of how the VCE improved their physical assessment skills.

The majority of students $45.2 \%(n=19)$ reported, "Agree" that (VCE) improved their written communication skills, versus $40.5 \%$ $(n=17)$ of students reported "Neutral" for the (VCE) effects on improving their oral communication skills.

E-learning in nursing is crucial in the nursing curriculums. Using the VCE in the medical surgical course helped students correlate what they learned in theory lecture with the virtual patients' cases, and they had more chances to assess and implement medical surgical nursing care to virtual patients in a safe virtual setting. The VCE provided a digital learning venue for nursing students. Receptive instructions given to students helped students navigate in the virtual learning environment. VCE implementation depended highly on giving more directive online instructions. Conducting a computer-based online tutorial for VCE was helpful to familiarize students to VCE virtual online resources. Students learned how to navigate in the virtual hospital, assess virtual patients, check their labs, vital signs, review medical records, and administer medications. Students were encouraged to enter the virtual hospital, learn, try, make mistakes, and try again. VCE allowed them to manipulate in the virtual hospital without any physical damage on the virtual patients, and improved safe learning experience will less anxiety. The evaluation report provided by the end of the VCE training session provided summative and informative feedback to students. The virtual hospital provides online individualized interaction between the students and the virtual patients, and this will provide an effective online learning experience for the nursing students.

Lack of literature that investigated the effectiveness of VCE in nursing curriculums justified the need for this study, coupled with the approach to teaching millennials. Incorporating e-learning methodologies is of great importance as many nursing students' generational age falls within the Generation X (born during1963 -1980) or Millennial (born during 1980-2000). There is strong evidence that most Millennials are accustomed to and thereby comfortable with technology [8]. The emergence of Instagram, Snapchat, Facebook, and Twitter promotes even more "connectivity". A recent study reported that $96 \%$ of millennials belong to at least one social network [9]. The emergence of Facebook, Instagram, Twitter, and Snapchat serves as a vehicle for additional technological modes of communication. It is no wonder that our technology savvy culture may influence how students learn and approach curriculum [10]. Thus, facilitating the need to incorporate e-learning to improve student nurses' learning experiences

\section{Study Limitations and Strengths}

There are a few limitations in this study that warrant further discussion. First, the sample was not randomly selected. The sampling was based on convenience sampling method. And the study targeted only one course to evaluate the effectiveness of VCE. Future studies are highly recommended to investigate the VCE benefits for more nursing courses including the maternity and pediatrics courses. Second, the VCE has some concerns related to accessibility and affordability due to the need to have internet connection upon using VCE, moreover, accessibility issues are a concern for students who may have auditory or visual deficits since the software does not have audio captions.

The strength of this study included the integration of a diversity policy, the pedagogical strategies of using VCE in a theory course supported students' learning with multiple diverse cultural, ethnic, and gender backgrounds, the virtual hospital patients reflected multiple ethnicities, and students were from different ethnicities, this provided a fair and interactive learning experience for the students.

\section{Conclusion and Study Implications}

This study provided quantitative and qualitative self-reported findings related to utilizing VCE in nursing education. Findings are reported from nursing student's own perspective and reflects the VCE efficacy in improving their learning experiences, their physical assessment skills, and communication skills. The VCE can be beneficial in providing a safe self-paced digital learning environment, in which students agreed in general that it improved their learning experiences in the targeted nursing program.

The virtual clinical excursions (VCE) provide online access to a virtual hospital that aligns with the work environment in which students are expected to work in after graduation. Integrating the virtual clinical excursions in the nursing courses will provide students with more flexibility in using the online learning resources at the students' convenience, and students will have more motivation to learn new concepts and virtually administer medications to patients. 
This study has implications for the nursing education, and the researchers highly recommend nursing faculty to integrate virtual e-learning systems, in this study VCE digital learning platform have utilized and investigated for its effectiveness, and nursing students agreed overall that been beneficial in improving their learning experiences. Evaluation of VCE effectiveness in improving the learning experiences of students is integral to improve the quality of nursing education, and may be particularly helpful in pre-licensure and online nursing programs.

\section{Acknowledgement}

We would like to thank the students who participated in this study.

\section{Funding Statement}

This work was supported by the Chancellor's Office, California State University, CRT, 2015. No conflict of interest is present at any point of this study.

\section{References}

1. American Association of Colleges of Nursing (2012) New AACN Data Show an Enrollment Surge in Baccalaureate and Graduate Programs Amid Calls for More Highly Educated Nurses, Press release.

2. Abuatiq A (2017) Nursing Students Experience With Virtual Clinical Excursions. Indianapolis. Indiana, USA.

3. Rodriguez SM (2013) The Impact of Limited Clinical Sites on Pre licensure Nursing Education Programs: Current Issues and Recommendations for the Future. Master of Arts in Nursing Scholarly Projects: 73.
4. Giddens J, Fogg L, Carlson Sabelli L (2010) Learning and engagement with a virtual community by undergraduate nursing students. Nurs Outlook 58(5): 261-267.

5. Giddens J, Hrabe D, Carlson-Sabelli L, Fogg, L, North S (2012) The impact of a virtual community on student engagement and academic performance among baccalaureate nursing students. J Prof Nurs 28(5): 284-290.

6. Secco L, Wilson K, Bulman D (2013) Pilot Evaluation of a Virtual Clinical Excursion (VCE) Among Senior Nursing Students: Part 1 - Quantitative Findings. Canadian Journal of Nursing Informatics 7(3\&4).

7. Gonzalez L, Fenske C (2012) Use of a Virtual Community to Contextualize Learning Activities. J Nurs Edu 51(1): 38-41.

8. Pardue KT, Morgan P (2008) Millennials considered: A new generation, new approaches, and implications for nursing education. Nurs Educ Perspect 29(2): 74-79.

9. Childs RD, Gingrich G, Piller M (2009) The future workforce: Gen Y has arrived. Public Manager 38(4): 21.

10. Chelliah J, Clarke E (2011) Collaborative teaching and learning: overcoming the digital divide. On the Horizon 19(4): 276-285.

11. MacIntyre RC, Murray TA, Teel CS, Karshmer JF (2009) Five recommendations for pre licensure clinical nursing education. J Nurs Edu 48(8): 447-453.

12. Sharon L Lewis, Shannon Ruff Dirksen, Margaret M, Heitkemper, Linda Bucher (2012) Virtual Clinical Excursion 3.0 Publisher Elsevier Health Sciences.
Creative Commons Attribution 4.0 International License

For possible submissions Click Here
Submit Article

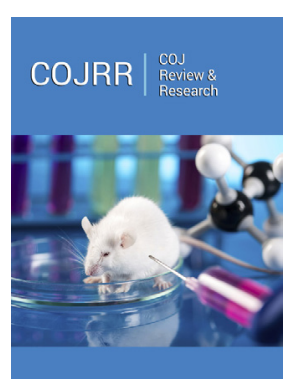

COJ Reviews \& Research

Benefits of Publishing with us

- High-level peer review and editorial services

- Freely accessible online immediately upon publication

- Authors retain the copyright to their work

- Licensing it under a Creative Commons license

- Visibility through different online platforms 